This paper is available online at http://www.math.nthu.edu.tw/tjm/

\title{
PERIODICITY IN MUTUALISM SYSTEMS WITH IMPULSE
}

\author{
Dan Ye and Meng Fan
}

\begin{abstract}
Easily verifiable sufficient criteria are established for the existence of periodic solutions of two mutualism systems with impulse. The approach is based on the coincidence degree and its related continuation theorem.
\end{abstract}

\section{INTRODUCTION}

The dynamic relationship between species has long been and will continue to be one of the dominant themes in both ecology and mathematical ecology due to its theoretical and practical significance. Many authors have devoted themselves to this topic $[2-5,7-14,16-21,23-25]$. But most of these work restricts to predator-prey system $[4,5,10,13,16,17,21]$ and competition systems[2, 3, 7, 12, 18-20], little has been done for mutualism systems [8, 9, 24, 25].

Recently, some authors devote themselves to the study of impulsive differential equation[1, 15, 22, 26]. However, in the study of the dynamic relationship between species, the effect of some impulsive factors has been ignored, which exists widely in the real world. For example, we notice that the births of many species are not continuous but happen at some regular time(For instance, the births of some wildlife are seasonal). It is reasonable to regard the births of species at these time as impulse to the species. Moreover, the human beings have been harvesting or stocking species at some time, then the species is affected by another type of impulse. One can conceive that such factors have great impact on the growth of a population. If we incorporate these impulsive factors into the models of population interactions, the models must be governed by impulsive ordinary differential equations. However, such systems, especially mutualism systems are rarely studied in the literature. So

Received December 30, 2003; accepted January 27, 2005.

Communicated by Sze-Bi Hsu.

2000 Mathematics Subject Classification: 34K45, 34K13, 92D25.

Key words and phrases: Positive periodic solutions, Mutualism systems, Coincidence degree, Impulse. Supported by the National Natural Science Foundation of P. R. China (No. 10201005). 
in this paper, we focus our attention on the existence of periodic solutions of two mutualism systems with impulse, i.e.,

$$
\begin{aligned}
y_{i}^{\prime}(t)= & y_{i}(t)\left[-d_{i}(t)-\frac{y_{i}(t)}{a_{i}(t)+b_{i}(t) y_{j}(t)}-c_{i}(t) y_{i}(t)\right], \\
& t \neq t_{k}, k=1,2 \cdots \\
\triangle y_{i}(t)= & y_{i}\left(t^{+}\right)-y_{i}\left(t^{-}\right)=\left(b_{i k}+h_{i k}\right) y_{i}(t), t=t_{k}, \\
& y_{i}(0)=y_{i 0}, i, j=1,2, i \neq j
\end{aligned}
$$

and

$$
\begin{aligned}
y_{i}^{\prime}(t)= & y_{i}(t)\left[-d_{i}(t)-a_{i i}(t) y_{i}(t)+a_{i j}(t) y_{j}(t)\right], \quad t \neq t_{k}, k=1,2 \cdots \\
\triangle y_{i}(t)= & y_{i}\left(t^{+}\right)-y_{i}\left(t^{-}\right)=\left(b_{i k}+h_{i k}\right) y_{i}(t), \quad t=t_{k}, \\
& y_{i}(0)=y_{i 0}, i, j=1,2, i \neq j,
\end{aligned}
$$

where

- $b_{i k}$ : the birth rate of $y_{i}$ at time $t_{k}$;

- $h_{i k}$ : the harvesting (stocking) rate of $y_{i}$ at time $t_{k}$. When $h_{i k}<0$, it stands for harvesting, while $h_{i k}>0$ means stocking;

- $d_{i}(t)$ : the death rate of $y_{i}(t)$;

- $a_{i}(t)$ : the carrying capacity of $y_{i}$ at time $t$ when the other species is absent;

- $c_{i}(t)$ and $a_{i i}(t)$ : the intraspecies competition coefficient of $y_{i}$ at time $t$;

- $b_{i}(t)$ and $a_{i j}(t)(i \neq j)$ : the mutualism coefficients;

- $y_{i}\left(t_{k}^{+}\right)$and $y_{i}\left(t_{k}^{-}\right)$represent the right and the left limit of $y_{i}$ at $t_{k}$, respectively. In this paper, it is assumed that $y_{i}$ is left-continuous at $t_{k}$.

System (1.2) is the standard model for the mutualism of two species. System (1.1) is a model for mutualism proposed by R. May, where it is assumed that the carrying capacity of one species is a increasing function of the other species.

In (1.1) and (1.2), we assume that

$\left(A_{1}\right) b_{i k} \geq 0, b_{i k}+h_{i k} \geq 0$, and $a_{i}(t), b_{i}(t), c_{i}(t), d_{i}(t), a_{i j}(t)(i, j=1,2)$ are nonnegative continuous $\omega$-periodic functions;

$\left(A_{2}\right)$ there exists a positive integer $q$, such that $t_{k+q}=t_{k}+\omega, b_{i(k+q)}=b_{i k}, h_{i(k+q)}=$ $h_{i k}$. Without loss of generality, we also assume that if $t_{k} \neq 0$ and $[0, \omega] \bigcap\left\{t_{k}\right\}=$ $t_{1}, t_{2} \cdots t_{m}$, then it follows that $q=m$.

It is trivial to show that the solutions of (1.1) and (1.2) with positive initial value remain positive too. Making the change of variables

$$
y_{i}(t)=e^{x_{i}(t)}, i=1,2 \text {. }
$$


then systems (1.1) and (1.2) are reformulated as the following, respectively.

$$
\begin{aligned}
x_{1}^{\prime}(t) & =-d_{i}(t)-\frac{e^{x_{i}(t)}}{a_{i}(t)+b_{i}(t) e^{x_{j}(t)}}-c_{i}(t) e^{x_{i}(t)}, t \neq t_{k}, k=1,2 \cdots \\
\triangle x_{i}(t) & =x_{i}\left(t^{+}\right)-x_{i}\left(t^{-}\right)=\ln \left(1+b_{i k}+h_{i k}\right), t=t_{k}, \\
& x_{i}(0)=\ln \left\{y_{i 0}\right\}>0, i=1,2, i \neq j .
\end{aligned}
$$

and

$$
\begin{aligned}
x_{1}^{\prime}(t) & =-d_{i}(t)-a_{i i}(t) e^{x_{i}(t)}+a_{i j}(t) e^{x_{j}(t)}, t \neq t_{k}, k=1,2 \cdots \\
\triangle x_{i}(t) & =x_{i}\left(t^{+}\right)-x_{i}\left(t^{-}\right)=\ln \left(1+b_{i k}+h_{i k}\right), t=t_{k}, \\
& x_{i}(0)=\ln \left\{y_{i 0}\right\}>0, i=1,2, i \neq j .
\end{aligned}
$$

For (1.1) and (1.2), we have similar lemma and definitions. So we only relate such results for (1.1).

Lemma 1.1. If $y(t)=\left(y_{1}(t), y_{2}(t)\right)$ is a positive $\omega$ periodic solution of (1.1), then $x_{i}(t)=\ln \left\{y_{i}(t)\right\}$ is an $\omega$-periodic solutions of (1.3), and vice versa.

Definition 1.1. The mapping $x:[0, \omega] \longrightarrow R^{2}$ is called a solution of system (1.3) in $[0, \omega]$, if

(i) $x(t)$ is partly continuous, $\left\{t_{k}\right\} \cap[0, \omega]$ are discontinuous points of the first kind of $x(t)$ and left continuous.

(ii) $x(t)$ satisfies system $(1.3)$ in $[0, \omega]$.

Definition 1.2. The mapping $x: R \longrightarrow R^{2}$ is called an $\omega$-periodic solution of system (1.3), if

(i) $x(t)$ is a solution of $(1.3)$ in $[0, \omega]$.

(ii) $x(t)$ satisfies $x(t+\omega-0)=x(t-0), t \in R$.

Obviously, if $x(t)$ is a solution of (1.3) or (1.4) satisfying $x(0)=x(\omega)$ in $[0, \omega]$, then from the periodicity of the vector field of (1.3) or (1.4), we know that the function

$$
x^{*}(t)=\left\{\begin{array}{l}
x(t-j \omega), t \in[j \omega,(j+1) \omega] \\
x^{*}(t) \text { is left continuous at } t_{k} .
\end{array}\right.
$$

is an $\omega$-periodic solution of (1.3) or (1.4). So, in order to achieve the existence of periodic solution for (1.3) or (1.4), it is sufficient to find the solutions of (1.3) 
or (1.4) in $[0, \omega]$ satisfying $x(0)=x(\omega)$, that is, to find solutions of the following equations in $[0, \omega]$

$$
\begin{aligned}
x_{1}^{\prime}(t)= & -d_{i}(t)-\frac{e^{x_{i}(t)}}{a_{i}(t)+b_{i}(t) e^{x_{j}(t)}}-c_{i}(t) e^{x_{i}(t)}, t \neq t_{k}, k=1,2 \cdots \\
\triangle x_{i}(t)= & x_{i}\left(t^{+}\right)-x_{i}\left(t^{-}\right)=\ln \left(1+b_{i k}+h_{i k}\right), t=t_{k}, \\
& x_{i}(0)=x_{i}(\omega)>0, i=1,2, i \neq j .
\end{aligned}
$$

or

$$
\begin{aligned}
x_{1}^{\prime}(t)= & -d_{i}(t)-a_{i i}(t) e^{x_{i}(t)}+a_{i j}(t) e^{x_{j}(t)}, t \neq t_{k}, k=1,2 \cdots \\
\triangle x_{i}(t)= & x_{i}\left(t^{+}\right)-x_{i}\left(t^{-}\right)=\ln \left(1+b_{i k}+h_{i k}\right), t=t_{k}, \\
& x_{i}(0)=x_{i}(\omega)>0, i=1,2, i \neq j .
\end{aligned}
$$

For simplicity and convenience in the following discussion, we will use the following notations throughout the paper

$$
\bar{f}:=\frac{1}{\omega} \int_{0}^{\omega} f(t) d t, f^{u}=\sup _{t \in[0, \omega]} f(t), f^{l}=\inf _{t \in[0, \omega]} f(t),
$$

where $f$ is an $\omega$-periodic function.

\section{Existence of Periodic Solution}

In order to obtain the existence of positive periodic solution of (1.1) or (1.2), for the readers' convenience, we shall present below a few of concepts and results from [6], which will be basic for this section.

Let $X, Z$ be normed vector spaces, $L: D o m L \subset X \rightarrow Z$ be a linear mapping, $N: X \rightarrow Z$ be a continuous mapping. The mapping $L$ will be called a Fredholm mapping of index zero if $\operatorname{dimKer} L=\operatorname{codim} \operatorname{Im} L<+\infty$ and $\operatorname{Im} L$ is closed in $Z$. If $L$ is a Fredholm mapping of index zero and there exist continuous projectors $P: X \rightarrow X$ and $Q: Z \rightarrow Z$ such that $\operatorname{ImP}=\operatorname{Ker} L, \operatorname{ImL}=\operatorname{Ker} Q=$ $\operatorname{Im}(I-Q)$, it follows that $L \mid \operatorname{Dom} L \cap \operatorname{KerP}:(I-P) X \rightarrow \operatorname{Im} L$ is invertible. We denote the inverse of that map by $K_{P}$. If $\Omega$ is an open bounded subset of $X$, the mapping $N$ will be called $L$-compact on $\bar{\Omega}$ if $Q N(\bar{\Omega})$ is bounded and $K_{P}(I-Q) N: \bar{\Omega} \rightarrow X$ is compact. Since $\operatorname{Im} Q$ is isomorphic to $\operatorname{Ker} L$, there exists an isomorphism $J: \operatorname{Im} Q \rightarrow \operatorname{Ker} L$.

Lemma 2.1. (Continuation Theorem) Let L be a Fredholm mapping of index zero and $N$ be L-compact on $\bar{\Omega}$. Suppose 
(a) For each $\lambda \in(0,1)$, every solution $x$ of $L x=\lambda N x$ is such that $x \notin \partial \Omega$;

(b) $Q N x \neq 0$ for each $x \in \partial \Omega \cap K e r L$ and

$$
\operatorname{deg}\{J Q N, \Omega \cap \operatorname{Ker} L, 0\} \neq 0 .
$$

Then the operator equation $L x=N x$ has at least one solution lying in DomL $\cap \bar{\Omega}$.

Let

$$
\begin{aligned}
& C\left[0, \omega ; t_{1}, t_{2}, \ldots t_{m}\right] \\
& =\left\{\begin{array}{l|l}
x:[0, \omega] \rightarrow R^{2} & \begin{array}{l}
x(t) \text { is continuous with respect to } t \neq t_{1}, \ldots, t_{m} ; \\
x(t+0) \text { and } x(t-0) \text { exist at } t_{1}, \ldots, t_{m} ; \\
x\left(t_{k}\right)=x\left(t_{k}-0\right), k=1,2, \ldots, m
\end{array}
\end{array}\right\} .
\end{aligned}
$$

Define

$$
\frac{1}{\omega} \sum_{k=1}^{q} \ln \left(1+b_{i k}+h_{i k}\right)-\bar{d}_{i}:=\triangle_{i} .
$$

Now we are ready to attack the existence of positive periodic solution of (1.1).

Lemma 2.2. Assume that $\triangle_{1}>0, \triangle_{2}>0$, then the system of algebraic equations

$$
\begin{aligned}
& \triangle_{1}-\frac{v_{1}}{\bar{a}_{1}+\bar{b}_{1} v_{2}}-\bar{c}_{1} v_{1}=0, \\
& \triangle_{2}-\frac{v_{2}}{\bar{a}_{2}+\bar{b}_{2} v_{1}}-\bar{c}_{2} v_{2}=0,
\end{aligned}
$$

has a unique positive solution $v=\left(v_{1}^{*}, v_{2}^{*}\right)^{T}$.

Proof. Consider the function

$$
f\left(v_{2}\right)=\triangle_{2}-\frac{v_{2}}{\bar{a}_{2}+\bar{b}_{2} \frac{\triangle_{1}\left(\bar{a}_{1}+\bar{b}_{1} v_{2}\right)}{\bar{c}_{1}\left(\bar{a}_{1}+\bar{b}_{1} v_{2}\right)+1}}-\bar{c}_{2} v_{2} .
$$

One can easily see that

$$
f(0)=\triangle_{2}>0, f\left(\frac{\triangle_{2}}{\bar{c}_{2}}\right)=-\frac{\frac{\triangle_{2}}{\bar{c}_{2}}}{\bar{a}_{2}+\bar{b}_{2} \frac{\triangle_{1}\left(\bar{a}_{1}+\bar{b}_{1} \frac{\triangle_{2}}{\bar{c}_{2}}\right)}{\bar{c}_{1}\left(\bar{a}_{1}+\bar{b}_{1} \frac{\triangle_{2}}{\bar{c}_{2}}\right)+1}}<0,\left.\frac{d f}{d v_{2}}\right|_{v_{2} \geq 0}<0,
$$


then from the zero point theorem and the monotonicity of $f\left(v_{2}\right)$, it follows that there exists a unique $v_{2}^{*} \in\left(0, \frac{\triangle_{2}}{\bar{c}_{2}}\right)$ such that $f\left(v_{2}^{*}\right)=0$ and then $v_{1}^{*}=\frac{\triangle_{1}\left(\bar{a}_{1}+\bar{b}_{1} v_{2}^{*}\right)}{\bar{c}_{1}\left(\bar{a}_{1}+\bar{b}_{1} v_{2}^{*}\right)+1}>$ 0 . The proof is complete.

Theorem 2.1. Assume that $\left(A_{1}\right),\left(A_{2}\right)$ hold. Moreover, if $\triangle_{1}>0, \triangle_{2}>0$ and $\bar{b}_{1} \bar{b}_{2} \triangle_{1} \triangle_{2} \neq 1$, then system (1.1) has at least one positive $\omega$ periodic solution.

Proof. Let

$$
X=\left\{x=\left(x_{1}, x_{2}\right)^{T} \in C\left[0, \omega ; t_{1}, \ldots t_{m}\right] \mid x(\omega)=x(0)\right\}, Z=X \times R^{2 q} .
$$

Define

$$
\|x\|_{C}=\sup _{t \in[0, \omega]}|x|,\|z\|_{Z}=\|x\|_{C}+\|y\|, x \in X, y \in R^{2 q},
$$

where $|\cdot|$ is any norm of $R^{2}$ and $\|\cdot\|$ is any norm of $R^{2 q}$. Then it is trivial to check that $X, Z$ are both Banach spaces when they are endowed with the above norm $\|\cdot\|_{C}$ and $\|\cdot\|_{Z}$, respectively.

Let

$$
\begin{aligned}
& \operatorname{dom} L \subset X\left\{x=\left(x_{1}, x_{2}\right)^{T} \in C\left[0, \omega ; t_{1}, \cdots t_{m}\right] \mid x(\omega)=x(0)\right\}, \\
& L: \operatorname{dom} L \rightarrow Z, L x=\left(x^{\prime}, \triangle x\left(t_{1}\right) \ldots \triangle x\left(t_{q}\right)\right), \\
& N: X \rightarrow Z \\
& \quad N x=\left(\left(W_{i}(t)\right)_{2 \times 1},\left(\ln \left(1++b_{i k}+h_{i k}\right)\right)_{2 \times q}\right)
\end{aligned}
$$

where

$$
W_{i}(t)=-d_{i}(t)-\frac{e^{x_{i}(t)}}{a_{i}(t)+b_{i}(t) e^{x_{j}(t)}}-c_{i}(t) e^{x_{i}(t)}, i, j=1,2, i \neq j .
$$

Then

$$
\begin{aligned}
\operatorname{Ker} L & =\left\{x: x=A \in R^{2}, t \in[0, \omega]\right\} \\
\operatorname{ImL} & =\left\{z=\left(f, C_{1} \cdots C_{q}\right) \in Z: \int_{0}^{\omega} f(s) d s+\sum_{k=1}^{q} C_{k}=0\right\}
\end{aligned}
$$

and

$$
\operatorname{dim} \operatorname{Ker} L=2=\operatorname{codim} \operatorname{Im} L .
$$

Since $I m L$ is closed in $Z, L$ is a Fredholm mapping of index zero. Let

$$
\begin{aligned}
& P x=\frac{1}{\omega} \int_{0}^{\omega} x(t) d t \\
& Q z=Q\left(f, C_{1} \cdots C_{q}\right)=\left(\frac{1}{\omega}\left[\int_{0}^{\omega} f(s) d s+\sum_{k=1}^{q} C_{k}\right], 0 \cdots 0\right) .
\end{aligned}
$$


It is easy to show that $P, Q$ are continuous projectors such that

$$
\operatorname{ImP}=\operatorname{Ker} L, \operatorname{Im} L=\operatorname{Ker} Q=\operatorname{Im}(I-Q) .
$$

Furthermore, the generalized inverse (to $L$ ) $K_{P}: \operatorname{ImL} \rightarrow \operatorname{Dom} L \bigcap \operatorname{KerP}$ exists.

Now, we derive the explicit expression for $K_{P}$. Let $z=\left(f, C_{1} \ldots C_{q}\right) \in \operatorname{ImL}$, then $x \in X$ satisfies

$$
\begin{aligned}
& x^{\prime}(t)=f(t), t \neq t_{k}, k=1,2 \ldots \\
& \left.\triangle x(t)\right|_{t=t_{k}}=C_{k} .
\end{aligned}
$$

Then

$$
x(t)=\int_{0}^{t} f(s) d s+\sum_{t>t_{k}} C_{k}+x(0) .
$$

Note that $x(t) \in \operatorname{Ker} P$, i.e., $\frac{1}{\omega} \int_{0}^{\omega} x(s) d s=0$. From (2.1), we get

$$
\int_{0}^{\omega} \int_{0}^{t} f(s) d s d t+\int_{0}^{\omega} \sum_{t>t_{k}} C_{k} d t+\omega x(0)=0
$$

and hence

(2.2) $x(t)=\int_{0}^{t} f(s) d s+\sum_{t>t_{k}} C_{k}-\frac{1}{\omega} \int_{0}^{\omega} \int_{0}^{t} f(s) d s d t-\sum_{t=1}^{q} C_{k}+\frac{1}{\omega} \sum_{t=1}^{q} C_{k} t_{k}$,

that is,

(2.3) $K_{P} z=\int_{0}^{t} f(s) d s+\sum_{t>t_{k}} C_{k}-\frac{1}{\omega} \int_{0}^{\omega} \int_{0}^{t} f(s) d s d t-\sum_{t=1}^{q} C_{k}+\frac{1}{\omega} \sum_{t=1}^{q} C_{k} t_{k}$.

Thus

$$
\begin{aligned}
& Q N x=\left(\left(\frac{1}{\omega} \int_{0}^{\omega} W_{i}(s)+\sum_{k=1}^{q} \ln \left(1+b_{i k}+h_{i k}\right)\right)_{2 \times 1},(0, \cdots 0)_{2 \times 1}\right) \\
K_{P}(I-Q) N x & \\
= & \left(\int_{0}^{t} W_{i}(s) d s+\sum_{t>t_{k}}^{q} \ln \left(1+b_{i k}+h_{i k}\right)\right)_{2 \times 1} \\
& \left.-\left(\left(\frac{t}{\omega}-\frac{1}{2}\right)\left(\int_{0}^{\omega} W_{i}(s) d s+\sum_{k=1}^{q} \ln \left(1+b_{i k}+h_{i k}\right)\right)\right)_{2 \times 1}^{q} \ln \left(1+b_{i k}+h_{i k}\right)-\frac{1}{\omega} \sum_{k=1}^{q} \ln \left(1+b_{i k}+h_{i k}\right) t_{k}\right)_{2 \times 1} \\
& -\left(\frac{1}{\omega} \int_{0}^{\omega} \int_{0}^{t} W_{i}(s) d s d t+\sum_{k=1}\right.
\end{aligned}
$$


Obviously, $Q N$ and $K_{P}(I-Q) N$ are continuous. It is trivial to show that that $\overline{K_{P}(I-Q) N(\bar{\Omega})}$ is compact for any open bounded set $\Omega \subset X$. Moreover, $Q N(\bar{\Omega})$ is bounded. Thus, $N$ is $L$-compact on $\bar{\Omega}$ with any open bounded set $\Omega \subset X$.

Now we ready to search for an appropriate open, bounded subset $\Omega$ for the application of the continuation theorem. Corresponding to the operator equation $L x=\lambda N x, \lambda \in(0,1)$, we have

$$
\begin{aligned}
x_{i}^{\prime}(t) & =\lambda\left[-d_{i}(t)-\frac{e^{x_{i}(t)}}{a_{i}(t)+b_{i}(t) e^{x_{j}(t)}}-c_{i}(t) e^{x_{i}(t)}\right], t \neq t_{k}, k=1,2 \cdots \\
\triangle x_{i}(t) & =x_{i}\left(t^{+}\right)-x_{i}\left(t^{-}\right)=\lambda \ln \left(1+b_{i k}+h_{i k}\right), t=t_{k}, \\
x_{i}(0) & =x_{i}(\omega), i=1,2, i \neq j .
\end{aligned}
$$

Suppose that $x \in X$ is a solution of system (2.4) for a certain $\lambda \in(0,1)$. Integrating on both sides of (2.4) from 0 to $\omega$, we obtain

$$
\int_{0}^{\omega}\left[-d_{i}(t)-\frac{e^{x_{i}(t)}}{a_{i}(t)+b_{i}(t) e^{x_{j}(t)}}-c_{i}(t) e^{x_{i}(t)}\right] d t+\sum_{k=1}^{q} \ln \left(1+b_{i k}+h_{i k}\right)=0
$$

That is,

$$
\int_{0}^{\omega} \frac{e^{x_{i}(t)}}{a_{i}(t)+b_{i}(t) e^{x_{j}(t)}} d t+\int_{0}^{\omega} c_{i}(t) e^{x_{i}(t)} d t=\triangle_{i} \omega
$$

It follows from (2.4) and (2.5) that

$$
\begin{aligned}
\int_{0}^{\omega}\left|x_{i}^{\prime}(t)\right| d t \leq & \bar{d}_{i} \omega+\int_{0}^{\omega}\left[\frac{e^{x_{i}(t)}}{a_{i}(t)+b_{i}(t) e^{x_{j}(t)}}+c_{i}(t) e^{x_{i}(t)}\right] d t \\
& +\sum_{k=1}^{q} \ln \left(1+b_{i k}+h_{i k}\right) \\
= & 2 \sum_{k=1}^{q} \ln \left(1+b_{i k}+h_{i k}\right) .
\end{aligned}
$$

Since $x \in X$, there exist $\xi_{i} \in[0, \omega]$, such that

$$
x_{i}\left(\xi_{i}\right)=\min _{t \in[0, \omega]} x_{i}(t), i=1,2 .
$$

On the other hand, note that $\sup x_{i}(t)$ exists and there exist $\eta_{i} \in[0, \omega]$ such that $t \in[0, \omega]$

$$
x_{i}\left(\eta_{i}^{+}\right)=\sup _{t \in[0, \omega]} x_{i}(t), i=1,2 .
$$


If $\eta_{i} \neq t_{k}$, then $x_{i}\left(\eta_{i}^{+}\right)=x_{i}\left(\eta_{i}\right)$ while if $\eta_{i}=t_{k}$, we have $x_{i}\left(\eta_{i}^{+}\right)=x_{i}\left(t_{k}^{+}\right)$.

From (2.5) and (2.7), we obtain

$$
\triangle_{i} \omega \geq \int_{0}^{\omega} c_{i}(t) e^{x_{i}\left(\xi_{i}\right)} d t=\bar{c}_{i} \omega e^{x_{i}\left(\xi_{i}\right)},
$$

and hence,

$$
x_{i}\left(\xi_{i}\right) \leq \ln \left\{\frac{\triangle_{i}}{\bar{c}_{i}}\right\} .
$$

From (2.6) and (2.9), we obtain

(2.10) $\left.x_{i}(t) \leq x_{i}\left(\xi_{i}\right)+\int_{0}^{\omega}\left|x_{i}^{\prime}(t)\right| d t<\ln \left\{\frac{\triangle_{i}}{\bar{c}_{i}}\right\}+2 \sum_{k=1}^{q} \ln \left(1+b_{i k}+h_{i k}\right)\right):=M_{i}$,

By (2.5) and (2.8), we also have

$$
\begin{gathered}
\int_{0}^{\omega} c_{i}(t) e^{x_{i}\left(\eta_{i}^{+}\right)} d t+\overline{\left(\frac{1}{a_{i}}\right)} e^{x_{i}\left(\eta_{i}^{+}\right)} \omega \geq \triangle_{i} \omega \\
\left(\overline{\left(\frac{1}{a_{i}}\right)}+\bar{c}_{i}\right) e^{x_{i}\left(\eta_{i}^{+}\right)} \geq \triangle_{i}
\end{gathered}
$$

and hence,

$$
x_{i}\left(\eta_{i}^{+}\right) \geq \ln \left\{\frac{\triangle_{i}}{\overline{\left(\frac{1}{a_{i}}\right)}+\bar{c}_{i}}\right\} .
$$

From (2.6) and (2.11), we have

$$
\begin{aligned}
x_{i}(t) \geq & x_{i}\left(\eta_{i}^{+}\right)-\int_{0}^{\omega}\left|x_{i}^{\prime}(t)\right| d t \geq \ln \left\{\frac{\triangle_{i}}{\overline{\left(\frac{1}{a_{i}}\right)}+\bar{c}_{i}}\right\} \\
& \left.-2 \sum_{k=1}^{q} \ln \left(1+b_{i k}+h_{i k}\right)\right)=\overline{M_{i}}
\end{aligned}
$$

which, together with (2.10), implies

$$
\sup _{t \in[0, \omega]}\left|x_{i}(t)\right|<\max \left\{\left|M_{i}\right|,\left|\overline{M_{i}}\right|\right\}:=N_{i} .
$$


Clearly, $N_{i}(i=1,2)$ are independent of $\lambda$.

By Lemma 2.2, it is easy to show

$$
\begin{aligned}
& \triangle_{1}-\frac{e^{x_{1}}}{\bar{a}_{1}+\bar{b}_{1} e^{x_{2}}}-\bar{c}_{1} e^{x_{1}}=0 \\
& \triangle_{2}-\frac{e^{x_{2}}}{\bar{a}_{2}+\bar{b}_{2} e^{x_{1}}}-\bar{c}_{2} e^{x_{2}}=0
\end{aligned}
$$

has a unique solution $x^{*}=\left(x_{1}^{*}, x_{2}^{*}\right)^{T}$ in Int $R^{2}$. Set $H=\left\|\left(N_{1}, N_{2}\right)^{T}\right\|+A$, where A is taken sufficiently large such that the unique solution of (2.13) satisfies $\left\|x^{*}\right\|=\left\|\left(x_{1}^{*}, x_{2}^{*}\right)^{T}\right\|<A$, and $\left\|x\left(t_{k}+0\right)\right\|<H, k=1,2 \cdots q$, then $\|x\|_{C}<H$.

Let $\Omega=\left\{x=\left(x_{1}, x_{2}\right)^{T} \in X \mid\left\|\left(x_{1}, x_{2}\right)^{T}\right\|_{C}<H\right\}$, then it is clear that $\Omega$ verifies the requirement $(a)$ of Lemma 2.1. When $x=\left(x_{1}, x_{2}\right)^{T} \in \partial \Omega \bigcap \operatorname{Ker} L=$ $\partial \Omega \bigcap R^{2}, x=\left(x_{1}, x_{2}\right)^{T}$ is a constant vector in $R^{2}$ with $\|x\|_{C}=\left\|\left(x_{1}, x_{2}\right)^{T}\right\|_{C}=$ $H$. Then

$$
Q N x=\left(\left(\begin{array}{c}
\triangle_{1}-\frac{e^{x_{1}}}{\bar{a}_{1}+\bar{b}_{1} e^{x_{2}}}-\bar{c}_{1} e^{x_{1}} \\
\triangle_{2}-\frac{e^{x_{2}}}{\bar{a}_{2}+\bar{b}_{2} e^{x_{1}}}-\bar{c}_{2} e^{x_{2}}
\end{array}\right),(0 \cdots 0)_{2 \times 1}\right) \neq 0 .
$$

In view of Theorem 2.1 and Lemma 2.2, from direct calculation, we get $\operatorname{deg}(J Q N$, $\Omega \bigcap \operatorname{Ker} L, 0) \neq 0$, where the degree is Brouwer degree, and the isomorphism $J$ of $\operatorname{Im} Q$ onto $\operatorname{Ker} L$ can be chosen to be the identity mapping, since $\operatorname{Im} Q=\operatorname{Ker} L$. By now we have proved that $\Omega$ verifies all requirements of Lemma 2.1, then $L x=$ $N x$ has at least one solution in $\operatorname{Dom} L \bigcap \bar{\Omega}$, i.e., (1.5) has at least one $\omega$ periodic solution in DomL $\bigcap \bar{\Omega}$, say $x=\left(x_{1}^{*}(t), x_{2}^{*}(t)\right)^{T}$. Set $y^{*}=\left(y_{1}^{*}(t), y_{2}^{*}(t)\right)^{T}=$ $\left(e^{x_{1}^{*}(t)}, e^{x_{2}^{*}(t)}\right)^{T}$, then $y^{*}=\left(y_{1}^{*}(t), y_{2}^{*}(t)\right)^{T}$ is one positive $\omega$ periodic solution of system (1.1). The proof is complete.

Remark 2.1. Theorem 2.1 tells us that, if the rate of the birth and the harvesting (stocking) is greater than the death rate, then (1.1) admits a positive $\omega$-periodic solution. This easily verifiable conditions are very reasonable since, otherwise, these species will extinct.

Next, we come to investigate the existence of positive periodic solution of (1.2).

Theorem 2.2. Assume $\left(A_{1}\right),\left(A_{2}\right)$ hold. Moreover, if $\triangle_{1}>0, \triangle_{2}>0$ and $a_{11}^{l} a_{22}^{l}>a_{21}^{u} a_{12}^{u}$, then system (1.2) has at least one positive $\omega$ periodic solution.

Proof. We define the same spaces and mapping as Theorem 2.1, except the mapping $\mathrm{N}$. Here, the mapping $N: X \rightarrow Z$ reads

$$
N x=\left(\left(-d_{i}(t)-a_{i i}(t) e^{x_{i}(t)}+a_{i j}(t) e^{x_{j}(t)}\right)_{2 \times 1},\left(\ln \left(1+b_{i k}+h_{i k}\right)\right)_{2 \times q}\right)
$$


Following similar arguments as in Theorem 2.1, one can easily prove that $N$ is $L$-compact on $\bar{\Omega}$ with any open bounded set $\Omega \subset X$.

Consider the operator equation $L x=\lambda N x, \lambda \in(0,1)$, i.e.,

$$
\begin{aligned}
x_{i}^{\prime}(t) & =\lambda\left[-d_{i}(t)-a_{i i}(t) e^{x_{i}(t)}+a_{i j}(t) e^{x_{j}(t)}\right], t \neq t_{k}, k=1,2 \cdots \\
\triangle x_{i}(t) & =x_{i}\left(t^{+}\right)-x_{i}\left(t^{-}\right)=\lambda \ln \left(1+b_{i k}+h_{i k}\right), t=t_{k}, \\
x_{i}(0) & =x_{i}(\omega), i=1,2, i \neq j .
\end{aligned}
$$

Suppose that $x \in X$ is a solution of system (2.14) for a certain $\lambda \in(0,1)$. Integrating on both sides of (2.14) from 0 to $\omega$, we obtain

$$
\int_{0}^{\omega}\left[-d_{i}(t)-a_{i i}(t) e^{x_{i}(t)}+a_{i j}(t) e^{x_{j}(t)}\right] d t+\sum_{k=1}^{q} \ln \left(1+b_{i k}+h_{i k}\right)=0,
$$

That is,

$$
\triangle_{i} \omega+\int_{0}^{\omega} a_{i j}(t) e^{x_{j}(t)} d t=\int_{0}^{\omega} a_{i i}(t) e^{x_{i}(t)} d t
$$

It follows from $(2.14)$ and $(2.15)$ that

$$
\begin{aligned}
\int_{0}^{\omega}\left|x_{i}^{\prime}(t)\right| d t \leq & \bar{d}_{i} \omega+\int_{0}^{\omega} a_{i j}(t) e^{x_{j}(t)} d t+\int_{0}^{\omega} a_{i i}(t) e^{x_{i}(t)} d t \\
& +\sum_{k=1}^{q} \ln \left(1+b_{i k}+h_{i k}\right) \\
= & 2\left(\int_{0}^{\omega} a_{i i}(t) e^{x_{i}(t)} d t+\bar{d}_{i} \omega\right) .
\end{aligned}
$$

From (2.15)

$$
a_{11}^{l} \int_{0}^{\omega} e^{x_{1}(t)} d t \leq \triangle_{1} \omega+a_{12}^{u} \int_{0}^{\omega} e^{x_{2}(t)} d t .
$$

On the other hand from (2.15) and (2.17)

$$
\begin{aligned}
a_{22}^{l} \int_{0}^{\omega} e^{x_{2}(t)} d t & \leq \triangle_{2} \omega+a_{21}^{u} \int_{0}^{\omega} e^{x_{1}(t)} d t \\
& \leq \triangle_{2} \omega+\frac{a_{21}^{u}}{a_{11}^{l}}\left(\triangle_{1} \omega+a_{12}^{u} \int_{0}^{\omega} e^{x_{2}(t)} d t\right),
\end{aligned}
$$

then

$$
\left(a_{11}^{l} a_{22}^{l}-a_{21}^{u} a_{12}^{u}\right) \int_{0}^{\omega} e^{x_{2}(t)} d t \leq\left(a_{21}^{u} \triangle_{1}+a_{11}^{l} \triangle_{2}\right) \omega
$$


that is,

$$
\int_{0}^{\omega} e^{x_{2}(t)} d t \leq \frac{a_{21}^{u} \triangle_{1}+a_{11}^{l} \triangle_{2}}{a_{11}^{l} a_{22}^{l}-a_{21}^{u} a_{12}^{u}}
$$

which, together with (2.15), implies

$$
\frac{\triangle_{2} \omega}{a_{22}^{u}} \leq \int_{0}^{\omega} e^{x_{2}(t)} d t \leq \frac{a_{21}^{u} \triangle_{1}+a_{11}^{l} \triangle_{2}}{a_{11}^{l} a_{22}^{l}-a_{21}^{u} a_{12}^{u}}
$$

Similarly,

$$
\frac{\triangle_{1} \omega}{a_{11}^{u}} \leq \int_{0}^{\omega} e^{x_{1}(t)} d t \leq \frac{a_{12}^{u} \triangle_{2}+a_{22}^{l} \triangle_{1}}{a_{11}^{l} a_{22}^{l}-a_{21}^{u} a_{12}^{u}}
$$

From (2.16), (2.19) and (2.20), we get

$$
\begin{aligned}
\int_{0}^{\omega}\left|x_{i}^{\prime}(t)\right| d t & \leq 2\left(\int_{0}^{\omega} a_{i i}(t) e^{x_{i}(t)} d t+\bar{d}_{i} \omega\right) \\
& \leq 2\left(a_{i i}^{u} \frac{a_{i j}^{u} \triangle_{j}+a_{j j}^{l} \triangle_{i}}{a_{11}^{l} a_{22}^{l}-a_{21}^{u} a_{12}^{u}}+\bar{d}_{i} \omega\right) \\
& :=C_{i} i, j=1,2, i \neq j .
\end{aligned}
$$

Since $x \in X$, from (2.19) and (2.20)

$$
x_{i}^{l} \leq \ln \left\{\frac{a_{i j}^{u} \triangle_{j}+a_{j j}^{l} \triangle_{i}}{a_{11}^{l} a_{22}^{l}-a_{21}^{u} a_{12}^{u}}\right\}:=H_{i}, x_{i}^{u} \geq \ln \left\{\frac{\triangle_{1} \omega}{a_{11}^{u}}\right\}:=\bar{H}_{i},
$$

hence

$$
\begin{gathered}
x_{i}(t) \leq x_{i}^{l}+\int_{0}^{\omega}\left|x_{i}^{\prime}(t)\right| d t \leq H_{i}+C_{i}, \\
x_{i}(t) \geq x_{i}^{u}-\int_{0}^{\omega}\left|x_{i}^{\prime}(t)\right| d t \geq \bar{H}_{i}-C_{i}, \\
\sup _{t \in[0, \omega]}\left|x_{i}(t)\right|<\max \left\{\left|H_{i}+C_{i}\right|+1,\left|\bar{H}_{i}-C_{i}\right|+1\right\}:=D_{i} .
\end{gathered}
$$

Clearly, $D_{i}$ is independent of $\lambda$.

In view of Theorem 2.2, algebraic equations

$$
\triangle_{1}-\bar{a}_{11} e^{x_{1}}+\bar{a}_{12} e^{x_{2}}=0, \triangle_{2}-\bar{a}_{22} e^{x_{2}}+\bar{a}_{21} e^{x_{1}}=0
$$

have a unique solution $x^{*}=\left(x_{1}^{*}, x_{2}^{*}\right)^{T} \in R_{2}$. Set $D=\left\|\left(D_{1}, D_{2}\right)^{T}\right\|+B$, where B is taken sufficiently large such that the unique solution of (2.24) satisfies $\left\|x^{*}\right\|=$ $\left\|\left(x_{1}^{*}, x_{2}^{*}\right)^{T}\right\|<B$, and $\left\|x\left(t_{k}+0\right)\right\|<D, k=1,2 \cdots q$, then $\|x\|_{C}<D$. 
Let $\Omega=\left\{x=\left(x_{1}, x_{2}\right)^{T} \in X \mid\left\|\left(x_{1}, x_{2}\right)^{T}\right\|_{C}<D\right\}$, then it is clear that $\Omega$ verifies the requirement $(a)$ of Lemma 2.1. When $x=\left(x_{1}, x_{2}\right)^{T} \in \partial \Omega \bigcap \operatorname{KerL}=$ $\partial \Omega \bigcap R^{2}, x=\left(x_{1}, x_{2}\right)^{T}$ is a constant vector in $R^{2}$ with $\|x\|_{C}=\left\|\left(x_{1}, x_{2}\right)^{T}\right\|_{C}=$ $D$. Then

$$
\begin{aligned}
Q N x= & \left(\frac{1}{\omega}\left(\int_{0}^{\omega}\left[-d_{i}(t)-a_{i i}(t) e^{x_{i}}+a_{i j}(t) e^{x_{j}}\right)\right] d t\right. \\
& \left.\left.+\sum_{k=1}^{q} \ln \left(1+b_{i k}+h_{i k}\right)\right)_{2 \times 1},(0, \cdots 0)_{2 \times 1}\right) \\
= & \left(\left(\triangle_{i}-\bar{a}_{i i} e^{x_{i}}+\bar{a}_{i j} e^{x_{j}}\right)_{2 \times 1},(0, \cdots 0)_{2 \times 1}\right) \neq(0, \cdots 0)_{2 \times 1}
\end{aligned}
$$

In view of Theorem 2.2, from direct calculation, we get

$$
\begin{gathered}
\operatorname{deg}(J Q N, \Omega \bigcap \operatorname{Ker} L, 0)=\sum_{x^{*} \in Q N^{-1}(0)} \operatorname{sgn} J Q N\left(x^{*}\right), \\
J Q N\left(x^{*}\right)=\left|\begin{array}{ll}
-\bar{a}_{11} & \bar{a}_{12} \\
\bar{a}_{21} & -\bar{a}_{22}
\end{array}\right|>0,
\end{gathered}
$$

then $\operatorname{deg}(J Q N, \Omega \bigcap \operatorname{Ker} L, 0) \neq 0$, where the isomorphism $J$ of $\operatorname{Im} Q$ onto $\operatorname{Ker} L$ can be chosen to be the identity mapping, since $\operatorname{Im} Q=\operatorname{Ker} L$. By now we have proved that $\Omega$ verifies all requirements of Lemma 2.1, then $L x=N x$ has at least one solution in $\operatorname{Dom} L \bigcap \bar{\Omega}$, i.e., (1.6) has at least one $\omega$ periodic solution in $\operatorname{Dom} L \bigcap \bar{\Omega}$, say $x=\left(x_{1}^{*}(t), x_{2}^{*}(t)\right)^{T}$. Set $y^{*}=\left(y_{1}^{*}(t), y_{2}^{*}(t)\right)^{T}=\left(e^{x_{1}^{*}(t)}, e^{x_{2}^{*}(t)}\right)^{T}$, then $y^{*}=\left(y_{1}^{*}(t), y_{2}^{*}(t)\right)^{T}$ is one positive $\omega$ periodic solution of system (1.2). The proof is complete.

Remark 2.1. Theorem 2.2 tells us that, if the rate of the birth and the harvesting (stocking) is larger than the death rate and the effect of the intraspecies competition is greater than the mutualism then (1.2) admits a positive $\omega$-periodic solution. These conditions looks very reasonable.

\section{ACKNOWLEDGEMENT}

The authors are very grateful to the anonymous referees for their careful reading of the manuscript and excellent suggestions, which greatly improve the presentation of this paper and greatly motivate our future research.

\section{REFERENCES}

1. A. V. Anokhin, L. Berezansky and E. Braverman, Exponential stability of linear delay impulsive differential equations, J. Math. Anal. Appl., 193 (1995), 923-941. 
2. F, J, Ayala, M. E. Gilpin and J. G. Eherenfeld Competition between species: Theoretical models and experimental tests, Theoretical Population Biology, 4 (1973), $331-356$.

3. J. Chattopadhyay, Effect of toxic substances on a two-species competitive system, Ecol. Modelling, 84 (1996), 287- 289.

4. M. Fan and K. Wang, Global Existence of Positive Periodic Solutions of Periodic Predator Prey System with Infinite Delays, J. Math. Anal. Appl., 262(1) (2001), $1-11$.

5. M. Fan and K. Wang, Periodicity in a Delayed Ratio-Dependent Predator Prey System, J. Math. Anal. Appl., 262(1) (2001), 179-190.

6. R. E. Gaines and J. L. Mawhin, Coincidence degree and nonlinear differential equations, Springer-Verlag, Berlin, 1977.

7. M. E. Gilpin and F. J. Ayala, Global models of growth and competition, Proc. Nat. Acad. Sci. USA, 70 (1973), 3590-3593.

8. Goh B S. Stability in models of mutualism. Amer Natural, 1979. 113 261-275.

9. K. Gopalsamy and X. Z. He, Persistence, attractivity, and delay in facultative mutualism, J. Math Anal Appl, 215 (1997), 154-173.

10. S. B. Hsu and T. W. Huang, Global stability for a class of predator-prey systems, SIAM J. Appl. Math., 55(3) (1995), 763-783.

11. V. Hutson and R. Law, Permanent coexistence in general models of there interacting species, J. Math. Biol., 25 (1985), 285-298.

12. Z. Jin, The study for ecological and epidemical models influenced by impulses, Doctoral Dissertation, Xi' an Jiaotong Uiversity, 2001.

13. N. Kazarinov and P. van den Driessche, A model predator-prey systems with functional response, Math. Biosci., 39 (1978), 125-134.

14. Y. Kuang, Delay Differential Equations with Applications in Population Dynamics, Boston Academic Press, 1993.

15. V. Lakshmikantham, D. D. Bainov and P. S. Simeonov, Theory of Impulsive Differential Equations, World Scientific, Singapore, 1989.

16. P. H. Leslie and J. C. Gower, The properties of a stochastic model for the predatorprey type of interaction between two species, Biometrika, 47 (1960), 219-234.

17. Y. K. Li, Periodic solutions of periodic delay predator-prey system, Proc. Amer. Math. Soc., 12 (1999), 1331-1335.

18. Y. K. Li, Periodic solutions for delay Lotka-Volterra competition systems. J. Math. Anal. Appl., 246 (2000), 230-244.

19. X. Liao and J. Li, Stability in Gilpin-Ayala competition models with diffusion, Nonliear Anal. TMA, 28(10) (1997), 1751-1758. 
20. R. M. May anf W. J. Leonard, Nonlinear aspects of competition between three species, SIAM J. Appl. Math., 29 (1975), 243-253.

21. M. L. Rosenzweig and R. H. Macarthur, Graphical representation and stability conditions for predator-prey interactions, Amer. Naturalist, 47 (1963), 209-223.

22. J. H. Shen, On some asymptotic stability results of impulsive integro-differential equations, Chinese Math. Ann., 17A (1996), 759-765.

23. J. M. Smith, Models in Ecology, Cambridge Univ. Press, Cambridge, U.K., 1974.

24. C. L. Wolin and L. R. Lawlor, Model of facultative mutualism, Density effects. Amer Natura, 124 (1984), 843-862.

25. P. H. Yang and R. Xu, Golbal asymptotic stability of periodic solution in n-species cooperative system with time delays. J. Biomathematics, 13(6) (1998), 841-846.

26. J. S. Yu and B. G. Zhang, Stability theorems for delay differential equations with impulses, J. Math. Anal. Appl., 199 (1996), 162-175.

\section{Dan Ye}

School of Information Science and Engineering,

Northeast University,

Shenyang, Liaoning 110004,

P. R. China

\section{Meng Fan}

School of Mathematics and Statistics,

Northeast Normal University,

5268 Renmin Street,

Changchun, Jilin 130024,

P. R. China

E-mail: mfan@nenu.edu.cn 